\title{
Efeito do mentol e eugenol sobre as respostas fisiológicas do pacu Piaractus mesopotamicus
}

\section{Effect of menthol and eugenol on the physiological responses of pacu Piaractus mesopotamicus}

\author{
Milena Souza dos Santos Sanchez ${ }^{*}$; Robson Andrade Rodrigues ${ }^{2}$; \\ André Luiz Nunes ${ }^{3}$; Aline Mayra da Silva Oliveira ${ }^{4}$; Letícia Emiliani Fantini²; \\ Cristiane Meldau de Campos ${ }^{5}$
}

\begin{abstract}
Resumo
Foram avaliados o tempo de indução, o tempo de recuperação e as respostas fisiológicas de pacus Piaractus mesopotamicus submetidos a doses usuais de mentol e eugenol. Foram utilizados 56 indivíduos com peso total médio de $236,5 \pm 96,01 \mathrm{~g}$ e comprimento total médio de 22,8 $\pm 3,43 \mathrm{~cm}$, estes, expostos ao mentol $150 \mathrm{mg} \mathrm{L}^{-1}(\mathrm{n}=24)$, ao eugenol $100 \mathrm{mg} \mathrm{L}^{-1}(\mathrm{n}=24)$ e um grupo controle $(\mathrm{n}=8)$, não exposto a nenhum anestésico. Nos momentos 0,12 e 24 h após indução, foram realizadas colheitas sanguíneas nos peixes de cada tratamento $(\mathrm{n}=8)$ para avaliação das variáveis hematológicas e glicose plasmática. Os resultados foram submetidos à análise de variância $(\mathrm{P}<0,05)$ e as médias quando significativas foram comparadas pelo teste de Tukey $(\mathrm{P}<0,05)$. Ambos os anestésicos em suas respectivas concentrações induziram os peixes à anestesia cirúrgica. Não houve diferença significativa para o tempo de indução, ao contrário do tempo de recuperação que foi significativamente menor $(\mathrm{P}<0,05)$ para o mentol 150 $\mathrm{mg} \mathrm{L}^{-1}$. Não foi observado efeito dos tratamentos sobre os parâmetros hematológicos, com exceção dos valores de hemoglobina, os quais apresentaram efeito de interação entre os fatores, uma vez que os peixes anestesiados com mentol $150 \mathrm{mg} \mathrm{L}^{-1}$ apresentaram redução significativa $(\mathrm{P}<0,05)$ dos valores de hemoglobina $12 \mathrm{~h}$ após a indução, porém estes valores permaneceram inalterados $24 \mathrm{~h}$ após anestesia. Foi observado efeito de interação $(\mathrm{P}<0,01)$ entre os tratamentos e os tempos de amostragem para os níveis de glicose plasmática, que apresentaram redução significativa destes níveis $12 \mathrm{~h}$ após anestesia, e assim permaneceram $24 \mathrm{~h}$ após a indução anestésica. O mentol $150 \mathrm{mg} \mathrm{L}^{-1}$ e o eugenol $100 \mathrm{mg} \mathrm{L}^{-1}$ podem ser utilizados seguramente na indução anestésica de pacus durante a realização de biometrias.

Palavras-chave: Anestesia, biometria, estresse, hematologia
\end{abstract}

\footnotetext{
Abstract

The present study the aim was evaluate induction time, recovery time and physiological responses of pacus Piaractus mesopotamicus submitted to eugenol and menthol usual doses. Were used 56 pacus, which were exposed to menthol $150 \mathrm{mgL}^{-1}(\mathrm{n}=24)$ and eugenol $100 \mathrm{mgL}^{-1}(\mathrm{n}=24)$ and the control group

${ }^{1}$ Zootecnista, Discente do Curso de Mestrado em Zootecnia, Universidade Federal da Grande Dourados, UFGD, Dourados, MS. E-mail: milenasanchezzoo@hotmail.com

${ }^{2}$ Zootecnistas, Discentes do Curso de Mestrado em Zootecnia, Universidade Estadual de Mato Grosso do Sul, UEMS, Aquidauana, MS. E-mail: robzootecnia@live.com; leticia.emiliani@hotmail.com

${ }^{3}$ Zootecnista, M.e em Zootecnia, UEMS, Aquidauna, MS. E-mail: andre_lnunes@hotmail.com

${ }^{4}$ Zootecnista, Discente do Curso de Doutorado em Zootecnia, Universidade Estadual de Maringá, UEM, Maringá, PR. E-mail: alimayrazoo@yahoo.com.br

${ }_{5}^{5}$ Prof $^{\mathrm{a}} \mathrm{Dr}^{\mathrm{a}}$ em Aquicultura, UEMS, Aquidauana, MS. E-mail: cmeldau@uems.br
}

* Autor para correspondência 
$(\mathrm{n}=24)$ which doesn't were exposed to anesthetics. In the moment 0,12 and 24 hours after induction were performed collection of blood in fish $(n=8)$ for the hematologic parameters and plasma glucose evaluation. The recovery was conduced in aquariums free anesthetic, being induction and recovery times monitored. The results were submitted at analysis of variance $(\mathrm{P}<0.05)$ and the means when significant were compared by Tukey test $(\mathrm{P}<0.05)$. Both anesthetics in their respective concentrations induced fish to surgical anesthesia. There was no treatment effects on hematologic parameters, except for hemoglobin for which was observed interaction effect between factors, is significantly lower 150 $\mathrm{mgL}^{-1}$ showed significant reduction $(\mathrm{P}<0.05)$ of hemoglobin values 12 hours after induction, however these values remained unchanged 24 hours after anesthesia. Interaction effect $(\mathrm{P}<0.01)$ was observed between treatments and sampling times for the plasma glucose levels, because there was significant reduction in this levels 12 hours after anesthesia, and remained 24 hous after induction. Menthol 150 $\mathrm{mgL}^{-1}$ and eugenol $100 \mathrm{mgL}^{-1}$ can be used safely in the pacus induction during realization of biometrics.

Key words: Anesthesia, biometrics, stress, hematology

\section{Introdução}

O pacu Piaractus mesopotamicus é uma das espécies nativas brasileiras mais produzidas, por demonstrar alto desempenho produtivo na piscicultura (VIDAL et al., 2008). Os bons índices zootécnicos, rusticidade, excelência de sua carne e fácil adaptabilidade aos manejos e aos sistemas de produção intensiva, propiciam sua larga utilização na piscicultura (QUEIROZ et al., 2005).

Porserumpeixeagitado,oseumanejoédificultado, uma vez que apresentam excesso de movimentações durante a prática, o que pode ocasionar ferimentos e perda de escamas (GONÇALVES et al., 2008), aumentando a possibilidade de manifestação de doenças e desencadeando reações biológicas como estresse (BARBOSA; MORAES; INOUE, 2007). Essas alterações metabólicas são capazes de afetar o desempenho normal dos peixes, tornando o uso de anestésicos naturais uma alternativa para minimizar o efeito das intervenções nas funções vitais e fisiológicas desses animais (CUNHA, 2007).

Todos os anestésicos exigem concentrações e tempos distintos para induzirem ao estágio de anestesia, que estão associados às mudanças comportamentais visíveis. Dentre os anestésicos naturais produzidos no Brasil, encontramos o eugenol e o mentol, utilizados na substituição de anestésicos químicos, por serem eficientes, de baixo custo e de elevada disponibilidade no mercado interno (INOUE; SANTOS NETO; MORAES,
2003; INOUE; HACKBARTH; MORAES, 2004; CUNHA, 2007).

Em geral, as concentrações ideais dos anestésicos são determinadas em função do tempo de indução e de recuperação, por isso é comum o uso de anestésicos em função das classes de peso. Segundo informações coletadas em piscicultura, pacus que apresentam peso entre 200 a 300 g são anestesiados com doses de eugenol e mentol em torno de 100 a $150 \mathrm{mg} \mathrm{L}^{-1}$, respectivamente. De acordo com Deriggi, Inoue e Moraes (2006) e Inoue et al. (2011) a concentração de determinado anestésico não deve ser recomendada levando em consideração somente os tempos de indução e de recuperação, mas também, as respostas metabólicas sob os respectivos tempos.

Dessa forma, com o presente estudo o objetivo foi avaliar o tempo de indução, o tempo de recuperação e as respostas fisiológicas de pacus submetidos a doses usuais de mentol e eugenol.

\section{Material e Métodos}

O experimento e as análises foram conduzidos, respectivamente, no Laboratório de Sanidade de Organismos Aquáticos e no Laboratório de Ictioparasitologia da Universidade Estadual de Mato Grosso do Sul - UEMS, Unidade Universitária de Aquidauana. Pacus Piaractus mesopotamicus adquiridos de piscicultura comercial, foram 
estocados e aclimatados às condições experimentais durante sete dias em tanques de alvenaria com volume útil de 4.000 litros, com renovação constante de água, e alimentados com ração comercial extrusada (32\% de PB), duas vezes ao dia. Os peixes permaneceram em jejum cerca de 24 $\mathrm{h}$ antes do início do experimento.

Foram utilizados 56 pacus com peso total médio de $236,5 \pm 96,01 \mathrm{~g}$ e comprimento total médio de $22,8 \pm 3,43 \mathrm{~cm}$, distribuídos aleatoriamente em dois tratamentos e um grupo controle. O grupo controle foi constituído por um grupo de peixes submetido à colheita sanguínea antes da exposição aos anestésicos $(n=8)$. Os tratamentos corresponderam ao mentol na concentração de $150 \mathrm{mg} \mathrm{L}^{-1} \mathrm{e}$ ao eugenol na concentração de $100 \mathrm{mg} \mathrm{L}^{-1}$. A colheita de sangue foi realizada em três momentos distintos: $0 \mathrm{~h}, 12 \mathrm{~h}$ e $24 \mathrm{~h}$ após o momento da indução, sendo que a cada colheita sanguínea, oito peixes foram amostrados.

Os banhos de imersão, uma das formas mais utilizadas no uso de anestésicos no Brasil, foram realizados em aquários de vidro, contendo 10 litros de água, até que os peixes atingissem o estágio de anestesia cirúrgica proposto por Ross e Ross (1999). Para determinação da influência da concentração dos anestésicos no tempo de indução e no tempo de recuperação, os animais foram expostos individualmente a cada concentração, sendo o tempo monitorado por meio de cronômetro digital até o aparecimento dos padrões comportamentais avaliados.

Depois de anestesiados os peixes foram submetidos à colheita de sangue e biometria. Posteriormente, retornaram individualmente ao aquário livre de anestésico, para se determinar o tempo de recuperação, que consiste no retorno total do equilíbrio, como estabelecido por Hikasa et al. (1986). Peixes que tiveram seu sangue colhido $12 \mathrm{~h}$ e $24 \mathrm{~h}$ após a indução anestésica, também passaram pelo procedimento de biometria e sofreram manipulação simulando a colheita sanguínea $0 \mathrm{~h}$ após a indução anestésica.
Os peixes amostrados 12 e 24 h após a indução anestésica foram mantidos nos tanques de alvenaria até o momento da colheita de sangue. Os animais foram capturados e contidos com auxílio de um pano umedecido, com intuito de minimizar o estresse. Durante o experimento avaliou-se a mortalidade dos animais e também a concentração de oxigênio dissolvido e temperatura da água dos aquários, com oxímetro portátil YSI.

O sangue foi colhido por punção do vaso caudal, utilizando seringas e agulhas diferentes, em função do parâmetro a ser analisado. Para as análises hematológicas, o sangue foi colhido com seringas e agulhas umedecidas internamente com EDTA a 3\% e para análise de glicose plasmática as seringas foram umedecidas internamente com antiglicolítico (Glistab). Foi determinado o percentual de microhematócrito (GOLDENFARB et al., 1971), hemoglobina pelo método de cianometahemoglobina (COLLIER, 1944) e a contagem de eritrócitos em câmara de Neubauer. De posse desses dados, foram calculados os índices hematimétricos (WINTROBE, 1934), compreendidos pelo volume corpuscular médio (VCM) e concentração de hemoglobina corpuscular média (CHCM). A concentração de glicose plasmática foi determinada a partir de kit comercial colorimétrico (LabTest $\left.{ }^{\circledR}\right)$.

Para os tempos de indução e de recuperação o delineamento experimental adotado foi inteiramente casualizado com dois tratamentos (anestésicos) e oito repetições, sendo cada peixe considerado uma unidade experimental. Para os parâmetros hematológicos e glicose plasmática o delineamento experimental adotado foi inteiramente casualizado em esquema fatorial 3x3 (anestésicos x tempos de amostragem). Cada tratamento conteve oito repetições, sendo cada peixe considerado uma unidade experimental. Para todos os parâmetros avaliados os dados obtidos foram submetidos à análise de variância $(\mathrm{P}<0,05) \mathrm{e}$ quando significativos as médias foram comparadas pelo teste de Tukey $(\mathrm{P}<0,05)$. 


\section{Resultados e Discussão}

Durante os testes de indução e recuperação dos peixes, os parâmetros de oxigênio dissolvido e temperatura da água aferidos, apresentaram-se adequados para a produção de pacus, uma vez que a temperatura da água se manteve em 26,9 $\pm 0,07$ ${ }^{\circ} \mathrm{C}$ e o oxigênio dissolvido em 7,3 $\pm 0,28 \mathrm{mg} \mathrm{L}^{-1}$. De acordo com Mylonas et al. (2005) estes parâmetros não influenciam nos resultados, sugerindo que os anestésicos nas concentrações estudadas foram os principais responsáveis pelo tempo necessário de anestesia cirúrgica de pacus. Não foi observada mortalidade dos peixes anestesiados no período de até $24 \mathrm{~h}$ após a imposição dos estímulos experimentais.

No presente estudo, as concentrações de mentol $150 \mathrm{mg} \mathrm{L}^{-1}$ e de eugenol $100 \mathrm{mg} \mathrm{L}^{-1}$ utilizada nos banhos de imersão foram capazes de induzir todos os animais ao estágio de anestesia cirúrgica. Entretanto, os peixes submetidos à solução anestésica de eugenol apresentaram hiperatividade, observada pela euforia e rápida movimentação dentro do aquário, que diminuiu de acordo com a ação do anestésico. O mesmo comportamento foi observado em juvenis de pintado Pseudoplatystoma corruscans por Vidal et al. (2006) e em juvenis de matrinxã Brycon cephalus e tambaqui Colossoma macropomum por Vidal et al. (2007) submetidos a ação anestésica com eugenol.

De acordo com Hisano et al. (2008), estes efeitos também são observados para dourado Salminus brasiliensis quando submetidos a indução anestésica com óleo de cravo, cujo principio ativo é o eugenol.
No entanto, segundo Inoue, Afonso e Iwama (2005) as visíveis alterações comportamentais de juvenis de matrinxã com peso total de $76,4 \pm 5,5 \mathrm{~g}$ submetidos ao banho anestésico com eugenol $25 \mathrm{mg} \mathrm{L}^{-1}$ não foram prejudiciais, mostrando ser satisfatório o uso do eugenol para esta espécie, embora tenham apresentado comportamento adverso no primeiro contato com a solução anestésica.

Os efeitos observados neste estudo durante a exposição dos pacus ao eugenol $100 \mathrm{mg} \mathrm{L}^{-1}$, não foram observados nos peixes expostos à solução anestésica de mentol $150 \mathrm{mg} \mathrm{L}^{-1}$. Entretanto, os peixes apresentaram todos os estágios de anestesia até atingirem o estágio de anestesia cirúrgica, assim como verificado para o tambaqui por Façanha e Gomes (2005) e para juvenis de tilápia-do-nilo Oreochromis niloticus por Simões e Gomes (2009) quando trabalharam com o mentol para essas espécies. Sendo assim, Vidal et al. (2006) atribuem a hiperatividade dos peixes ao próprio eugenol.

O mentol $150 \mathrm{mg} \mathrm{L}^{-1}$ e o eugenol $100 \mathrm{mg} \mathrm{L}^{-1}$ mostraram-se eficientes para serem utilizados no manejo de pacu, uma vez que possibilitaram a anestesia cirúrgica com indução de 180 segundos e recuperação de 600 segundos, como recomendados por Park et al. (2009). Segundo esses autores a concentração ótima, não deve exceder esses tempos ao produzir ação anestésica. Na Tabela 1, pode-se observar que os tempos de indução apresentados pelos tratamentos, não diferem $(\mathrm{P}>0,05)$ estatisticamente entre si, diferente do tempo de recuperação o qual foi significativamente inferior $(\mathrm{P}<0,05)$ para o mentol $150 \mathrm{mg} \mathrm{L}^{-1}$.

Tabela 1. Tempo de indução e recuperação (média \pm erro-padrão) de pacus Piaractus mesopotamicus submetidos às concentrações usuais de mentol e eugenol.

\begin{tabular}{cccc}
\hline Tratamento & N & Indução (s) & Recuperação (s) \\
\hline Mentol 150 $\mathbf{~ m g ~ L}^{-1}$ & 8 & $71,13 \pm 9,78$ & $88,13 \pm 7,00^{\mathrm{b}}$ \\
Eugenol 100 $\mathbf{~ m g ~ L}^{-1}$ & 8 & $90,13 \pm 6,77$ & $117,38 \pm 7,32^{\mathrm{a}}$ \\
\hline
\end{tabular}

Médias de letras diferentes na mesma coluna diferem estatisticamente entre si pelo teste de Tukey $(\mathrm{P}<0,05)$.

Fonte: Elaboração dos autores. 
Resultados similares foram obtidos por Simões e Gomes (2009), que verificaram que o mentol na concentração de $150 \mathrm{mg} \mathrm{L}^{-1}$ é mais eficiente tanto na indução quanto na recuperação de juvenis de tilápia-do-nilo quando comparado a outras concentrações do mesmo anestésico. De acordo com Façanha e Gomes (2005) este mesmo efeito foi observado para tambaqui, juvenis de pacu (GONÇALVES et al., 2008) e dourado (PÁDUA et al., 2010). Entretanto, Vidal et al. (2006) ao induzirem juvenis de pintado com mentol observam tempo de indução e recuperação inferiores ao do presente trabalho.

Os resultados supracitados podem ser atribuídos ao estágio de anestesia profunda, o qual foi avaliado pelos autores ou ao peso dos peixes. Porém peixes da mesma espécie, mas de tamanhos diferentes exigem tempos distintos para que atinjam o estágio de anestesia e de recuperação desejado (ROSS e ROSS 2008). Entretanto, o tempo de recuperação pode ter sido influenciado pelo tempo de exposição ao anestésico (GOMES et al., 2001) e também pela ação do fármaco.

Os peixes possuem temperatura corporal igual àquela do ambiente onde vivem, cujo aumento, eleva a taxa metabólica e, por consequência, o consumo de oxigênio. Mesmo assim, os peixes compensam essa elevada demanda por oxigênio com maior número e/ou amplitude dos movimentos respiratórios, possibilitando que maior volume de água passe pelas brânquias, assim como as substâncias nela dissolvidas. Entretanto, no presente estudo, pode se afirmar que não houve influência da temperatura sobre os resultados, uma vez que esta foi à mesma para ambos os anestésicos testados (SCHMIDT-NILSEN, 2002).

A superfície de absorção é proporcionalmente maior em peixes menores, sendo assim, peixes menores absorvem mais anestésicos do que os peixes maiores e, consequentemente, seu tempo de indução anestésica é mais rápido e sua recuperação mais lenta (ROUBACH et al., 2002). Segundo Prince e Powell (2000) e Vidal et al. (2007) os resultados obtidos para o alto tempo de recuperação apresentado por peixes anestesiados com eugenol, são adequados para biometrias, cirurgias ou manejo de desova, visto que, nestas manipulações é necessário que os peixes permaneçam anestesiados por mais tempo, após serem retirados da solução anestésica.

Não foi observado efeito $(\mathrm{P}>0,05)$ dos tratamentos sobre os parâmetros hematológicos como hematócrito, número de eritrócitos, volume corpuscular médio (VCM) e concentração de hemoglobina corpuscular média (CHCM), como pode ser observado na Tabela 2. No entanto, observou-se efeito de interação $(\mathrm{P}<0,05)$ entre os fatores para os valores de hemoglobina, sendo que os peixes anestesiados com mentol $150 \mathrm{mg} \mathrm{L}^{-1}$ apresentaram redução significativa $(\mathrm{P}<0,05)$ nos valores de hemoglobina $12 \mathrm{~h}$ após a indução, porém esta, permaneceu inalterada $24 \mathrm{~h}$ após anestesia.

Essa alteração pode estar ligada à hiperatividade evidenciada nos peixes anestesiados com eugenol $100 \mathrm{mg} \mathrm{L}^{-1}$, considerando que nestas condições os animais demandam maior quantidade de oxigênio, a produção de hemoglobina aumenta para que a quantidade de oxigênio transportada para o sangue seja suficiente.

Segundo Morales et al. (2005), as mudanças nas respostas hematológicas de estresse, normalmente, não são observadas em exposições agudas, mas são comuns em exposições crônicas. Simões et al. (2012) ao utilizar óleo de cravo $250 \mathrm{mg} \mathrm{L}^{-1}$ na indução anestésica de juvenis de tilápia-donilo, observaram valores para concentração de hemoglobina significativamente menores $24 \mathrm{~h}$ após a indução anestésica. 
Tabela 2. Variáveis hematológicas (média \pm erro-padrão) de pacus Piaractus mesopotamicus submetidos às concentrações usuais de mentol e eugenol.

\begin{tabular}{|c|c|c|c|c|c|}
\hline \multirow[b]{2}{*}{ Tratamento } & \multicolumn{5}{|c|}{ Parâmetros Hematológicos } \\
\hline & $\begin{array}{c}\text { Hematócrito } \\
(\%)\end{array}$ & $\begin{array}{l}\text { Hemoglobina } \\
\left(\mathrm{g} \text { dL - }{ }^{-1}\right)\end{array}$ & $\begin{array}{l}\text { Eritrócito } \\
\left(\times 10^{6} \mu \mathrm{L}-1\right)\end{array}$ & $\begin{array}{c}\mathrm{VCM} \\
\text { (fL) }\end{array}$ & $\begin{array}{c}\text { CHCM } \\
\left(\text { g dL - }^{-1}\right)\end{array}$ \\
\hline \multicolumn{6}{|c|}{0 horas } \\
\hline Controle & $26,75 \pm 2,34$ & $9,45 \pm 1,08^{\mathrm{Aa}}$ & $1,36 \pm 0,14$ & $154,89 \pm 14,83$ & $33,56 \pm 3,20$ \\
\hline Mentol & $29,50 \pm 1,22$ & $9,75 \pm 0,54^{\mathrm{Aa}}$ & $1,39 \pm 0,11$ & $163,55 \pm 12,35$ & $29,60 \pm 2,44$ \\
\hline Eugenol & $29,13 \pm 0,69$ & $8,53 \pm 0,70^{\mathrm{Aa}}$ & $1,35 \pm 0,05$ & $168,49 \pm 5,57$ & $27,01 \pm 0,97$ \\
\hline \multicolumn{6}{|c|}{12 horas } \\
\hline Mentol & $29,13 \pm 1,33$ & $6,13 \pm 0,39^{\mathrm{bB}}$ & $1,29 \pm 0,11$ & $179,20 \pm 16,52$ & $18,14 \pm 0,71$ \\
\hline Eugenol & $28,13 \pm 1,13$ & $6,73 \pm 0,21^{\mathrm{bA}}$ & $1,47 \pm 0,17$ & $150,75 \pm 13,04$ & $21,13 \pm 1,11$ \\
\hline \multicolumn{6}{|c|}{24 horas } \\
\hline Mentol & $30,88 \pm 1,17$ & $7,72 \pm 0,44^{\mathrm{abAB}}$ & $1,65 \pm 0,10$ & $148,96 \pm 8,58$ & $22,17 \pm 1,82$ \\
\hline Eugenol & $27,25 \pm 1,91$ & $6,46 \pm 0,22^{\mathrm{bA}}$ & $1,66 \pm 0,11$ & $123,25 \pm 8,26$ & $22,41 \pm 1,95$ \\
\hline
\end{tabular}

Médias seguidas de letras minúsculas na mesma coluna indicam diferença significativa pelo teste de Tukey $(\mathrm{P}<0,05)$ entre os tratamentos dentro dos tempos de amostragem; Médias seguidas de letras maiúsculas indicam efeito de interação $(\mathrm{P}<0,05)$ entre os tratamentos e os tempos de amostragem; $\mathrm{VCM}=$ Volume Corpuscular médio; $\mathrm{CHCM}=$ Concentração de Hemoglobina Corpuscular Média; Mentol $150 \mathrm{mg} \mathrm{L}^{-1}$; Eugenol $100 \mathrm{mg} \mathrm{L}^{-1}$.

Fonte: Elaboração dos autores.

Segundo Wendelaar-Bonga (1997), as respostas de estresse desencadeiam alterações fisiológicas, classificadas como respostas primárias, secundárias e terciárias. Respostas primárias destacam-se pelo aumento na secreção de catecolaminas, adrenalina, noradrenalina e cortisol no plasma, sendo as respostas secundárias, as respostas metabólicas, como a alteração na glicemia, ácido lático, glicogênio hepático e muscular e as respostas hematológicas. As respostas terciárias são as alterações que levam à queda de desempenho produtivo, diminuindo a resistência dos peixes a doenças.

Foi observado efeito $(\mathrm{P}<0,01)$ do tempo de amostragem para os valores de glicose plasmática Oh após indução anestésica, visto que houve aumento significativo destes valores para os peixes expostos a ambos anestésicos. Porém, os animais expostos ao eugenol $100 \mathrm{mg} \mathrm{L}^{-1}$ apresentaram os maiores valores. Também foi observado efeito de interação $(\mathrm{P}<0,01)$ entre os tratamentos e os tempos de amostragem, pois houve redução significativa das concentrações plasmáticas $12 \mathrm{~h}$ após a indução, e assim permaneceram $24 \mathrm{~h}$ após a indução anestésica, como pode ser observado na Figura 1.
De acordo Simões et al. (2012), o uso de óleo de cravo $250 \mathrm{mg} \mathrm{L}^{-1}$ na indução anestésica de juvenis de tilápia-do-nilo aumenta significativamente as concentrações plasmáticas de glicose após a anestesia, quando comparado a outros tempos, porém os valores retornam para os níveis basais 6 $\mathrm{h}$ após a indução. A redução da glicose após $6 \mathrm{~h}$ da indução, também foi observada por Deriggi, Inoue e Moraes (2006) ao anestesiar a mesma espécie com eugenol 20 e $80 \mathrm{mg} \mathrm{L}^{-1}$.

Inoue et al. (2011) ao avaliar respostas metabólicas de tambaqui submetidos a banhos anestésicos com eugenol em concentrações de 20 e $60 \mathrm{mg} \mathrm{L}^{-1}$ por 15 minutos e amostrados 0 e $24 \mathrm{~h}$ após os banhos, observaram o aumento significativo nas concentrações plasmáticas de glicose $0 \mathrm{~h}$ após indução, sendo estes recuperados $24 \mathrm{~h}$ após os banhos. Resultados semelhantes foram encontrados por Barbosa, Moraes e Inoue (2007) para matrinxã em banhos de eugenol 15 e $60 \mathrm{mg} \mathrm{L}^{-1}$ por 10 minutos, e por Simões e Gomes (2009) para juvenis de tilápia-do-nilo quando submetidos a concentração de $250 \mathrm{mg} \mathrm{L}^{-1}$ de mentol por 9 minutos. 
Figura 1. Glicose plasmática (média \pm erro-padrão) de pacus Piaractus mesopotamicus submetidos ao mentol $150 \mathrm{mg} \mathrm{L}^{-1}$ e eugenol $100 \mathrm{mg} \mathrm{L}^{-1}$. Médias seguidas de letras minúsculas indicam diferença significativa entre os tratamentos dentro dos tempos de amostragem; Médias seguidas de letras maiúsculas indicam efeito de interação entre os tratamentos e os tempos de amostragem; Médias seguidas de letras diferentes diferem pelo teste de Tukey $(\mathrm{P}<0,05)$.

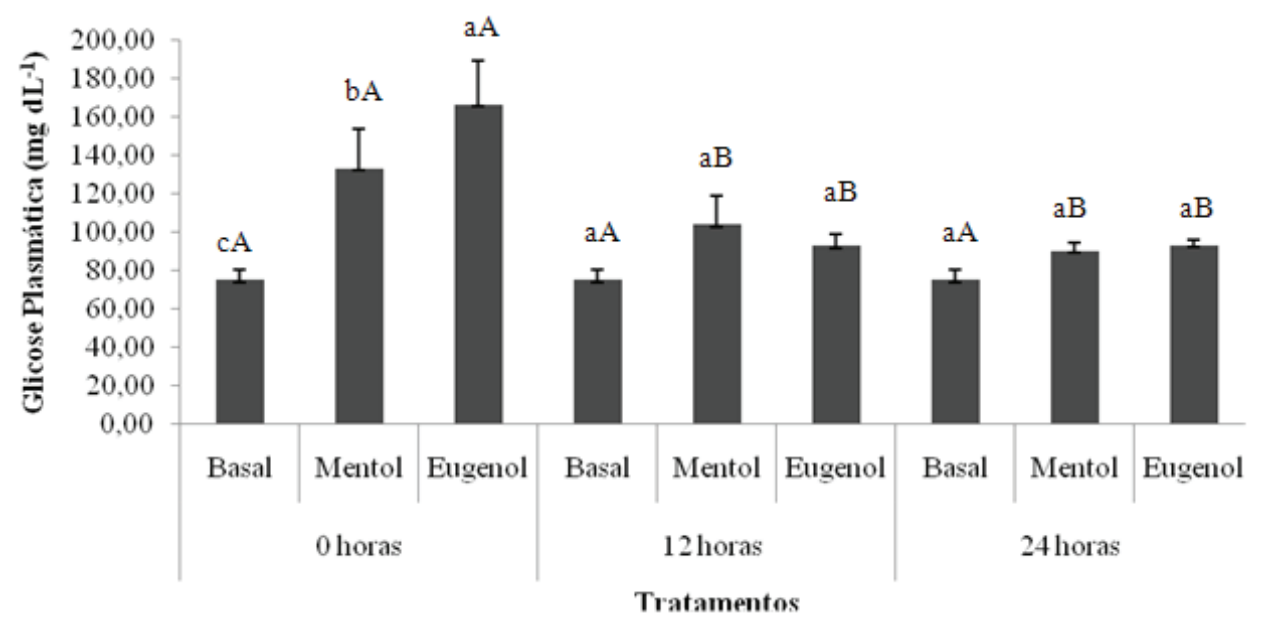

Fonte: Elaboração dos autores.

Desta forma pode se observar que a reação metabólica encontrada nos estudos supracitados é semelhante à observada neste estudo, podendo se afirmar que independente das condições de experimento, concentração e fármaco, as concentrações plasmáticas de glicose apresentadas pelos peixes sempre serão superiores no primeiro momento, ou seja, $0 \mathrm{~h}$ após a indução anestésica, voltando à normalidade posteriormente.

Elevações nas concentrações plasmáticas de glicose também foram observadas para carpa comum Cyprinus carpio por Velisek, Svobodova e Piackova (2005) e para a truta arco-íris oncorhynchus mykiss por Park et al. (2008), segundo os autores esse efeito pode ser atribuído ao anestésico eugenol e ao óleo de cravo, cujo principio ativo é o eugenol.

Segundo Simões et al. (2012), a mobilização de glicose, a partir do glicogênio estocado no fígado dos animais, ocorre sempre que há sinal externo de estresse.Aglicose pode serutilizada anaerobicamente como fonte de energia em situações onde há falta de oxigênio, diferente dos outros metabólitos. No entanto, o manuseio para aplicar o anestésico exige do animal uma reação muscular (comum em peixes) para a natação de arranque na tentativa de fuga, o que desencadeia a glicólise anaeróbica, requerendo maiores concentrações de glicose plasmática sem que haja alterações nas concentrações de cortisol.

Ao culminar a teoria proposta por Simões et al. (2012), Inoue et al. (2011), Barbosa, Moraes e Inoue (2007) e Deriggi, Inoue e Moraes (2006) com as respostas observadas neste estudo, observa-se que o uso dos valores de glicose associados ao tempo de indução e recuperação é pertinente, ou seja, suficiente para verificar que o uso de anestésicos na indução de peixes causam estresse adicional. Desta forma, devese considerar eficiente o anestésico que resulta em um menor tempo de recuperação dos peixes, uma vez que peixes anestesiados com quais quer que forem os anestésicos demonstram breves alterações fisiológicas nos períodos conseguintes a indução.

\section{Conclusões}

O mentol na concentração de $150 \mathrm{mg} \mathrm{L}^{-1} \mathrm{e}$ o eugenol na concentração de $100 \mathrm{mg} \mathrm{L}^{-1}$ podem ser utilizados seguramente na indução anestésica de pacus Piaractus mesopotamicus durante a realização de práticas de manejo como biometrias. 


\section{Agradecimentos}

À Universidade Estadual de Mato Grosso do Sul, à empresa Mar \& Terra e Piscicultura Nova Era pelo fornecimento dos peixes e ao Macroprograma Aquabrasil com apoio financeiro da Embrapa, MPA e CNPq.

\section{Referências}

BARBOSA, L. M.; MORAES, G.; INOUE, L. A. K. A. Metabolic responses of matrinxa to eugenol in anesthetic baths. Acta Scientiarum. Biological Sciences, Maringá, v. 29, n.3, p. 255-260, 2007.

COLLIER, H. B. The standardizations of blood haemoglobin determinations. Canadian Medical Association Journal, Vancouver, v. 50, n. 6, p. 550-552, 1944.

CUNHA, M. A. Anestesia em jundiás (Rhamdia quelen) expostos a substâncias isoladas de plantas. 2007. Dissertação (Mestrado em Zootecnia) - Universidade Federal de Santa Maria, Santa Maria.

DERIGGI, G.; INOUE, L. A. K. A.; MORAES, G. Stress responses in nile tilapia (Oreochromis niloticus): assessment of eugenol as na alternative anesthetic. Acta Scientiarum. Biological Sciences, Maringá, v. 28, n. 3, p. 269-274, 2006.

FAÇANHA, M. F.; GOMES, L. C. A eficácia do mentol como anestésico para tambaqui (Colossoma macropomum, Characiformes: Characidae). Embrapa Amazônia Ocidental, Manaus, v. 35, n. 1, p. 71-75, 2005.

GOLDENFARB, P. B.; BOWYER, F. P.; HALL, E.; BROSIOUS, E. Reproducibility in the hematology laboratory: the microhematocrit determinations. American Journal of Clinical Pathology, Maringá, v. 56, n. 1, p. 35-39, 1971.

GOMES, L. C.; CHIPPARI-GOMES, A. R.; LOPES, N. P. ROUBACH, R.; ARAUJO-LIMA, C. A. R. M. Efficacy of benzocaine as a anesthetic in juvenile tambaqui, Colossoma macropomum. Journal of the World Aquaculture Society, New Orleans, v. 32, n. 4, p. 426-431, 2001.

GONÇALVES, A. F. N.; SANTOS, E. C. C.; FERNANDES, J. B. K.; TAKAHASHI, L. S. Mentol e eugenol como substitutos da benzocaína na indução anestésica de juvenis de pacu. Acta Scientiarum. Biological Sciences, Maringá, v. 30, n. 3, p. 339-344, 2008.
HIKASA, Y.; TAKASE, K.; OGASAWARA, T.; OGASAWARA, S. Anaesthesia and recovery with tricane methanesulphonate, eugenol and thiopental sodium in the carp (Cyprinus carpio). Japanese Journal of Veterinary Science, Santa Maria, v. 48, n. 2, p. 341-351, 1986.

HISANO, H.; ISHIKAWA, M. M.; FERREIRA, R. A. BUlGarelli, A. L. A.; COSTA, T. R.; PÁDUA, S. B. Tempo de indução e de recuperação de dourados Salminus brasiliensis (Cuvier, 1816), submetidos a diferentes concentrações de óleo de cravo Eugenia sp. Acta Scientiarum. Biological Sciences, Maringá, v. 30, n. 3, p. 303-307, 2008.

INOUE, L. A. K. A.; AFONSO, L. O. B.; IWAMA, G. $\mathrm{K}$. Efeito do óleo de cravo na resposta de estresse do matrinxã (Brycon cephalus) submetidos ao transporte. Acta Amazônica, Manaus, v. 35, n. 2, p. 289-295, 2005.

INOUE, L. A. K. A.; BOIJINK, C. L.; RIBEIRO, P. T.; SILVA, A. M. D.; AFFONSO, E. G. Avaliação de respostas metabólicas do tambaqui expostos ao eugenol em banhos anestésicos. Acta Amazônica, Manaus, v. 41, n. 2, p. 327-332, 2011.

INOUE, L. A. K. A.; HACKBARTH, A.; MORAES, G. Avaliação dos anestésicos 2-phenoxyethanol e benzocaína no manejo do matrinxã Brycon cephalus (Gunther, 1869). Biodiversidade Pampeana, Uruguaiana, v. 2, n. 1, p. 10-15, 2004.

INOUE, L. A. K. A.; SANTOS NETO, C.; MORAES, G. Clove oil as anaesthetic for juveniles of matrinxã Brycon cephalus (Gunther, 1869). Ciência Rural, Santa Maria, v. 33, n. 5, p. 943-947, 2003.

MORALES, A. E.; CARDENETE, G.; ABELLÁN, E.; GARCÍA-REJÓN, L. Stress-related physiological responses to handling in common dentex (Dentex dentex Linnaeus, 1758). Aquaculture Research, Oxford, v. 36, n. 1, p. 33-40, 2005.

MYLONAS, C. C.; CARDINALETTI, G.; SIGELAKI, I.; POLZONETTI-MAGNI, A. Comparative efficacy of clove oil and 2-phenoxyethanol as anesthetics in the aquaculture of European sea bass (Dicentrarchus labrax) and gilthead sea bream (Sparus aurata) at different temperatures. Aquaculture, Amsterdam, v. 246, n. 1-4, p. 467-481, 2005.

PÁDUA, S. B.; PIETRO, P. S.; IGLESSIAS-FILHO, P. S.; ISHIKAWA, M. M.; HISANO, H. Mentol como anestésico para dourado (Salminus brasiliensis). Boletim do Instituto de Pesca, São Paulo, v. 32, n. 2, p. 143-148, 2010.

PARK, I. S.; PARK, M. O.; HUR, J. W.; KIM, D. S.; CHANG, Y. J.; KIM, Y. J.; PARK, J. Y.; JOHNSON, S. C. 
Anesthetic effects of lidocaine-hydrochloride on water parameters in simulated transport experiment of juvenile winter flounder, Pleuronectes americanus. Aquaculture, Amsterdam, v. 294, n. 1-2, p. 76-79, 2009.

PARK, M. O.; HUR, W. J.; IM, S. Y.; SEOL, D. W.; LEE, J.; PARK, I. S. Anaesthetic efficacy and physiological responses to clove oil anaesthetized kelp grouper Epinephelus bruneus. Aquaculture Research, Oxford, v. 39, n. 8, p. 877-884, 2008.

PRINCE, A.; POWELL, C. Clove oil as an anesthetic for invasive field procedures on adult rainbow trout. North American Journal of Fisheries Management, Bethesda, v. 20, n. 4, p. 1029-1032, 2000.

QUEIROZ, J. F.; LOURENÇO, J. N. P.; KITAMURA, P. C. SCORVO FILHO, J. D.; CYRINO, J. E. P.; CASTAGNOLLI, N.; VALENTI, W. C.; BERNARDINO, G. Aquaculture in Brazil: research priorities and potential for further international collaboration. World Aquaculture Magazine, v. 36, p. 45-50, 2005.

ROSS, L. G.; ROSS, B. Anaesthetic and sedative techniques for aquatic animals. 2. ed. Oxford: Blackwell Science, 1999, $159 \mathrm{p}$.

. Anaesthetic and sedative techniques for aquatic animals. 3. ed. Oxford: Blackwell Science, 2008, 240 p.

ROUBACH, R.; GOMES, L. C.; LOURENÇO, J. N. P.; FONSECA, F. A. L.; VAL, A. L. Efficacy of eugenol as anaesthetic for tambaqui Juvenile (Colossoma macropomum). In: VAL, A. L.; ALMEIDA-VAL, V. M. F.; McKINLEY, D. Tropical fish: news and reviews. Vancouver: International Congress on Biology of Fishes, 2002. p. 93-96.

SCHMIDT-NIELSEN, K. Fisiologia animal: adaptação e meio ambiente. 5. ed. São Paulo: Editora Santos, 2002. $600 \mathrm{p}$.
SIMÕES, L. N.; GOMES, L. C. Eficácia do mentol como anestésico para juvenis de tilápia do nilo. Arquivo Brasileiro de Medicina Veterinária e Zootecnia, Belo Horizonte, v. 61, n. 3, p. 613-620, 2009.

SIMÕES, L. N.; GOMIDE, A. T. M.; ALMEIDA-VAL, V. M. F.; VAL, A. L.; GOMES, L. C. O uso do óleo de cravo como anestésico em juvenis avançados de tilápia do Nilo (Oreochromis niloticus). Acta cientiarum. Animal Sciences, Maringá, v. 34, n. 2, p. 175-181, 2012.

VELISEK, J.; SVOBODOVA, Z.; PIACKOVA, V. Effect of clove oil anaesthesia on rainbow trout (Oncorhynchus mykiss). Acta Veterinaria Brno, v. 74, n. 1, p. 139-146, 2005.

VIDAL, L. V. O.; ALBINATI, R. C. B.; ALBINATI, A. C. L.; MECÊDO, G. R. Eugenol como anestésico de tilápiado-nilo. Pesquisa Agropecuária Brasileira, Brasília, v. 43, n. 8, p. 1069-1074, 2008.

VIDAL, L. V. O.; ALBINATI, R. C. B.; ALBINATI, A. C. L.; MECÊDO, G. R. Utilização do eugenol como anestésico para o manejo de juvenis de pintado (Pseudoplatystoma corruscans). Acta Scientiarum. Biological Sciences, Maringá, v. 28, n. 3, p. 275-279, 2006.

VIDAL, L. V. O.; FURUYA, W. M.; GRACIANO, T. S.; SCHAMBER, C. R.; SANTOS, L. D.; SOARES, C. M. Concentrações de eugenol para anestesia profunda e toxidade aguda em juvenis de piavuçu (Leporinus macrophalus). Acta Scientiarum. Biological Sciences, Maringá, v. 29, n. 4, p. 657-362, 2007.

WENDELAAR-BONGA, S. E. The stress response in fish. Physiological Review, Boston, v. 77, n. 3, p. 591625, 1997.

WINTROBE, M. M. Variation in the science and hemoglobin content of erythrocytes in the blood of various vertebrates. Folia Haematological, Philadelphia, v. 51, n. 32, p. 32-49, 1934. 
